\title{
Quantitative assessment of overall inflammatory bowel disease activity using labelled leucocytes: a direct comparison between indium-111 and technetium-99m HMPAO methods
}

Gastroenterology Unit and Department of Medical Physics, Royal Hallamshire Hospital, Sheffield

J C Mansfield

M H Giaffer

W B Tindale

C D Holdsworth

Correspondence to:

Gastroenterology Unit, Roya

Victoria Infirmary, Newcastle upon Tyne, NE2 4LP.

Accepted for publication 29 March 1995

\section{Abstract}

The ideal imaging method in inflammatory bowel disease would reliably detect inflammation, identify the correct intestinal location, and assess the severity of the disease. The aim of this study was to compare scintigraphic methods of quantifying overall disease activity using both indium111 (111 In) and technetium-99M (99mTc) HMPAO labelled leucocyte scans. The four day faecal excretion of ${ }^{111}$ In was measured after ${ }^{111}$ In scintigraphy in 24 patients known to have inflammatory bowel disease. The same patients also underwent ${ }^{99 \mathrm{~m}}$ Tc HMPAO scanning. The scans were performed 10 days or less apart with no changes in treatment between scans. Bowel activity on the ${ }^{99 \mathrm{~m}} \mathrm{Tc}$ HMPAO scans was assessed using a computer based method (scan score) and a visual grading method in a further 54 ${ }^{99 m}$ Tc HMPAO. The results showed a close correlation between inflammatory activity defined by faecal ${ }^{111}$ In excretion and the scan score generated from the computer analysis of the ${ }^{99 \mathrm{~m}}$ Tc HMPAO image (Spearman rank correlation: $\left.r_{s}=0.78 ; p<0.001\right)$. Accurate information to localise inflammatory activity could be obtained by simple visual assessment of both types of scan images, although image quality was superior with ${ }^{99 m}$ Tc HMPAO. Qualification of disease activity from 99mTc HMPAO images by visual grading was associated with a large variability, only $69 \%$ of scans had similar scores when graded by three observers. Computer generated image analysis was more reproducible. In conclusion, in inflammatory bowel disease ${ }^{99 \mathrm{~m}}$ Tc HMPAO scintigraphy and faecal ${ }^{111}$ In excretion correlated well. Either method can quantify and localise the inflammation. As ${ }^{99 m}$ Tc HMPAO scanning provides a quicker result, with a lower radiation dose, and avoids faecal collection, it may be the preferred method.

(Gut 1995; 37: 679-683)

Keywords: inflammatory bowel disease, 99m-technetium HMPAO, ${ }^{11} 1_{\text {indium, disease activity. }}$

\section{Introduction}

Both ${ }^{111}$ In tropolonate labelled leucocytes and ${ }^{99 \mathrm{~m}}$ Tc HMPAO labelled leucocytes have been successfully used to localise inflammatory bowel disease activity. ${ }^{1-5}$ Attempts to quantify the scan image into an index of overall disease activity, which could be used to monitor response to treatment, have been less successful. Clinical disease activity correlated well with a simple grading of the overall scan (zero to four $)^{2}$ but this was not sufficiently subtle to define changes before and after treatment. In the case of ${ }^{111}$ In scans the solution has been to measure the percentage of injected ${ }^{111}$ In excreted into the faeces over the following four days. This correlates well with other markers of disease activity and clinical indices. ${ }^{6}$ To quantify ${ }^{99 \mathrm{~m}}$ Tc HMPAO scans the bowel has been divided into regions, each region scored relative to isotope uptake in bone marrow, liver, and spleen and a score calculated by summation of the individual bowel regions. While segment by segment analysis has shown good correlations with endoscopic and histological grading of inflammatory activity, ${ }^{7}$ the overall scan activity index is less reliable than the scan's ability to localise inflammation, ${ }^{8}$ and suffers from interobserver variation. ${ }^{9}$

Computer assisted subtraction analysis has also been used to generate a scan score from ${ }^{99 m}$ Tc HMPAO scans. ${ }^{10}$ This aims to achieve reliable quantification of overall disease activity, similar to the faecal ${ }^{111}$ In excretion, and the speed and convenience of $99 \mathrm{mTc}$ HMPAO scintigraphy. Scan scores generated by this method correlate well with clinical disease activity and laboratory indices of active inflammation and have permitted patients with symptoms not caused by active inflammation to be identified. ${ }^{11}$

This study was designed to compare ${ }^{111}$ In and ${ }^{99 \mathrm{~m}} \mathrm{Tc}$ HMPAO scanning directly in the same patients, with particular attention to the methods of quantification. The objective was to determine the optimal method of quantitative leucocyte bowel scanning.

\section{Patients and methods}

Twenty four patients underwent both ${ }^{99 \mathrm{~m}} \mathrm{Tc}$ HMPAO and ${ }^{111}$ In labelled leucocyte scans. The second scan was performed within 10 days of the first and there were no changes in treatment between the scans. The $99 \mathrm{~m}$ Tc HMPAO scan was always performed before the ${ }^{111} \mathrm{In}$ scan.

The patients had a mean age of 43 years (range 17-68) and were equally distributed between male and female. The diagnosis, 
based on previous investigations, was Crohn's disease in 22 of the cases and ulcerative colitis in the remaining two. Six patients with Crohn's disease had undergone previous resective surgery, all surgery having been performed more than six months before the imaging. The disease duration, from diagnosis to scanning, ranged from three months to 22 years, mean eight years. The site of disease had not been fully determined by prior large and small bowel radiology in five patients, but was known to be limited to the colon in five, small bowel in eight, and present in both small and large bowel in six.

An additional $54^{99 \mathrm{~m}}$ Tc HMPAO scans, performed in the course of clinical assessment of disease activity, on 33 patients with Crohn's disease were analysed to compare the reproducibility of visual and computer assisted grading.

This study had the approval of the ethics committee of the former United Sheffield Hospitals and the Administration of Radioactive Substances Advisory Committee (ARSAC). The initial scan was clinically indicated to assess disease activity. Patients gave informed consent for the second (paired) scan. All scans were performed between February 1990 and January 1992.

\section{${ }^{111}$ Indium leucocyte labelling, imaging, and faecal collection}

Autologous leucocytes were separated and labelled with ${ }^{111}$ In tropolonate as previously described. ${ }^{12}$ In summary, $60 \mathrm{ml}$ of blood were taken into $5.5 \mathrm{ml}$ of acid citrate dextrose. Ten $\mathrm{ml}$ were centrifuged at $1500 \mathrm{~g}$ for 10 minutes to obtain cell free plasma. The erythrocytes were removed from the remaining blood by hydroxyethyl sedimentation at room temperature. The supernatant was then centrifuged to permit separation of the leucocytes from the platelets. After washing, the leucocytes were labelled in plasma with tropolone and ${ }^{111} \mathrm{In}$ chloride (Amersham International). Any free ${ }^{111}$ In was removed by a final wash in plasma, before reinjection. An average of $15 \mathrm{MBq}$ of ${ }^{111}$ In labelled cells was injected into each patient.

A dynamic study of the posterior lung fields was performed for 15 minutes after reinjection to exclude any cell injury during labelling. ${ }^{6}$

Abdominal scans were performed at three hours after reinjection using a large field of view gammacamera. Faecal ${ }^{111}$ In excretion was measured on a four day collection, which started immediately after the scan. All patients were given clear written and verbal instructions regarding the faecal collection. The importance of acquiring a complete four day collection was stressed. All faecal collections were done on an outpatient basis.

${ }^{99 \mathrm{~m}}$ Technetium HMPAO labelling and imaging The technique of labelling granulocytes in mixed leucocyte suspension using ${ }^{99 \mathrm{~m}} \mathrm{Tc}$ HMPAO has previously been described. ${ }^{13}$ Briefly, $102 \mathrm{ml}$ of venous blood were taken into $18 \mathrm{ml}$ of acid citrate dextrose. Twenty $\mathrm{ml}$ were centrifuged to obtain cell free plasma. Leucocytes were separated as described above and were resuspended in cell free plasma. Four $\mathrm{ml}$ of ${ }^{99 \mathrm{~m}} \mathrm{Tc} \mathrm{HMPAO}$, reconstituted according to the manufacturer's instructions, were added to the leucocyte suspension, mixed gently, and left for 10 minutes at room temperature. After this the labelled cells were washed once with plasma before being transferred to a heparinised plastic syringe for reinjection. An average of $200 \mathrm{MBq}$ of ${ }^{99 \mathrm{~m}} \mathrm{Tc}$ labelled cells was injected into each patient.

As with the ${ }^{111}$ In scintigraphy, a dynamic study of the posterior lung fields was carried out for the first 15 minutes after injecting the radiolabelled leucocytes. No patients showed prolonged lung retention of labelled cells.

Subsequently anterior and posterior abdominal scans were obtained at 40 minutes and 120 minutes using a large field of view gammacamera (IGE $400 \mathrm{~T}$ or Siemens ZLC 750 Digitrac), fitted with a low energy, high resolution collimator and interfaced to a microcomputer (Research Machines Nimbus PC20).

\section{Quantitative assessment of leucocyte scans}

Visual assessment - the ${ }^{99 \mathrm{~m}}$ Tc HMPAO scans acquired at 120 minutes were coded and scored blindly and independently by three physicians. A scoring system similar to that used by other investigators ${ }^{814}$ was used. The colon was divided into four regions (rectosigmoid, descending, transverse, and ascending) with a fifth region representing the small intestine. The imagine intensity in each region was compared with the distribution of isotope in the bone marrow (score 1), liver (score 2), and spleen (score 3 ). A score of 4 was assigned if the bowel intensity was greater than in the spleen. The addition of the scores for the five regions produced a visual grading score for the whole scan, with a potential range 0-20.

Computer generated scan score - using the image obtained at 120 minutes after reinjection of ${ }^{99 \mathrm{~m}} \mathrm{Tc}$ a computer generated scan score

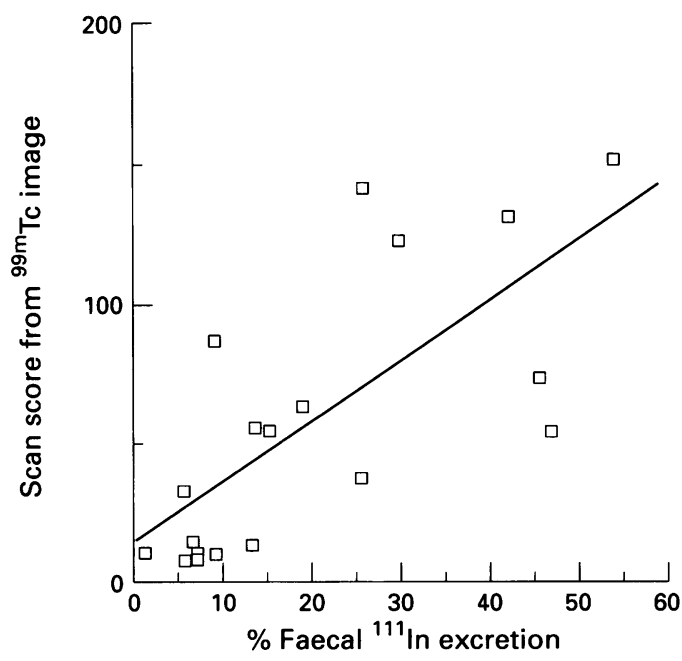

Figure 1: The computer generated scan score derived from the ${ }^{99 \mathrm{~m}}$ Tc HMPAO image $\mathrm{v}$ the percentage ${ }^{111}$ In faecal excretion in paired scans. 
was calculated. The method has been described previously. ${ }^{10}$ In outline, the patient image is scaled in size to that of a standard image, which consists of spleen, liver, and bone marrow activity without localised bowel uptake. The standard or 'background' image is then scaled in amplitude and digitally subtracted by computer from the patient's image, leaving only the bowel related uptake. The scan score, which is a dimensionless quantity, is then calculated from the counts in a $20 \%$ contour based on the maximum intensity in

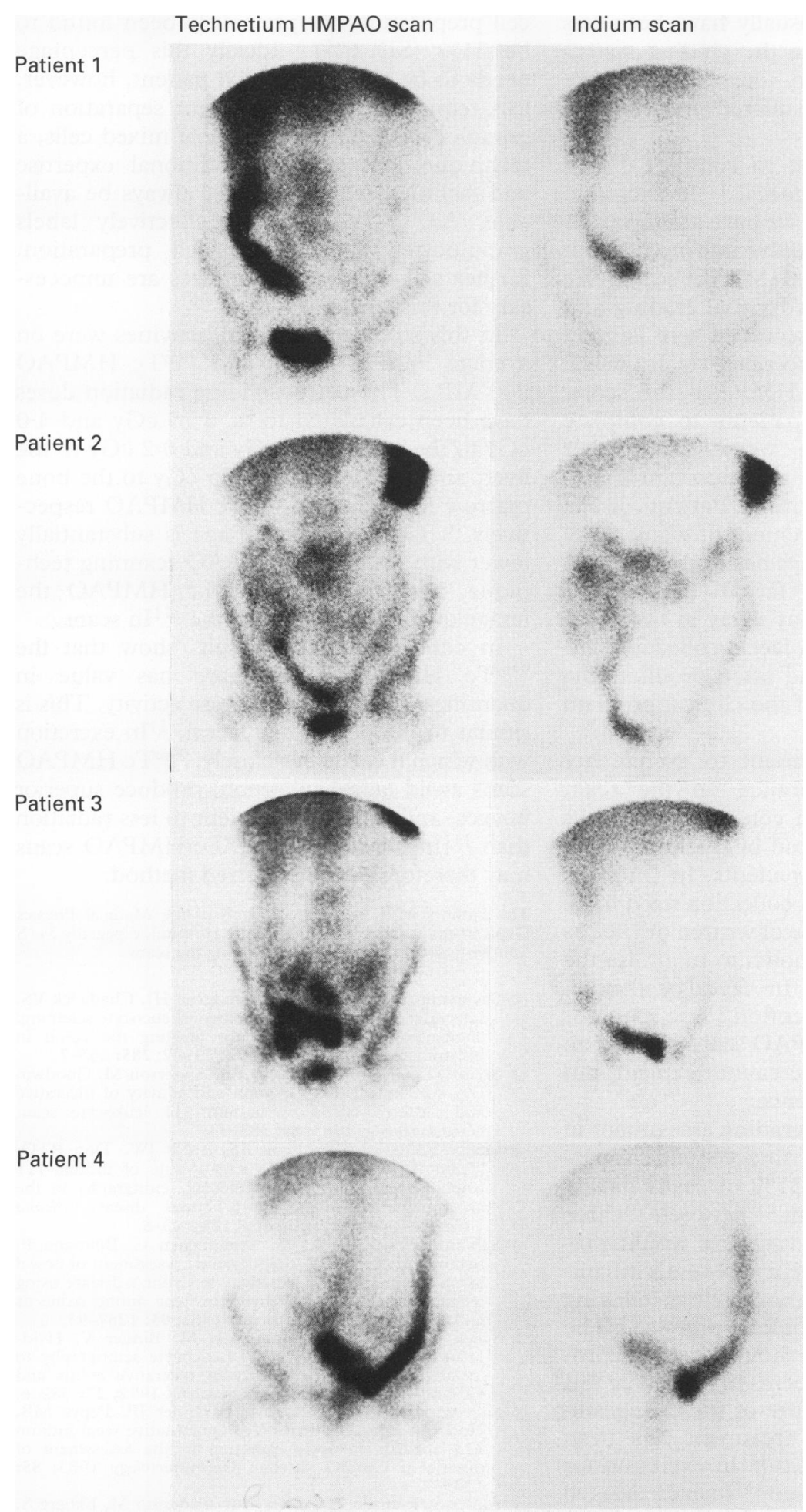

Figure 2: Four pairs of ${ }^{111}$ In and ${ }^{99 \mathrm{~m}} T c$ HMPAO images, showing the superior image quality of that obtained using ${ }^{99 \mathrm{~m}}$ Tc HMPAO. All the ${ }^{99 \mathrm{~m}}$ Tc HMPAO images were acquired at 120 minutes after injection. the abnormal gut. This value is corrected for image acquisition time and injected dose by normalisation to the bone marrow uptake of the scaled standard image.

Determination of percentage faecal excretion the indium activity excreted in the faeces was determined by counting each of the daily collections in a large volume sample gammacounter, equipped with a rotating table. The activity from all collections was summed and expressed as a percentage of the injected dose. As the percentage of labelling of granulocytes in the mixed cell suspension in each patient was not known, it was necessary to assume that this was constant across all subjects.

\section{Results}

Twenty four patients underwent paired ${ }^{99 \mathrm{~m}} \mathrm{Tc}$ HMPAO and ${ }^{111}$ In labelled scans. Three patients failed to complete their faecal collection despite verbal and written instructions of the procedure. A further patient became constipated during her four day collection after taking analgesia for a migraine attack. In this case the ${ }^{111}$ In leucocyte scan performed at two hours was at variance with the low faecal excretion. After the problem of her constipation became apparent this result was excluded from the analysis.

One patient had uptake on her ${ }^{99 \mathrm{~m}} \mathrm{Tc}$ HMPAO scan that did not conform to bowel outline. This remains an unexplained artefact. She had a low faecal ${ }^{111}$ In excretion $(2 \cdot 8 \%$ of injected dose) and a repeat ${ }^{99 \mathrm{~m} T \mathrm{~T}}$ HMPAO scan a few weeks later gave a correspondingly low scan score without repeat of the artefactual uptake. This is the only such artefact we have seen in over $200{ }^{99 \mathrm{~m}}$ Tc HMPAO bowel scans.

The remaining 19 paired ${ }^{99 \mathrm{~m}} \mathrm{Tc}$ HMPAO scan scores and percentage faecal ${ }^{111}$ In excretion results showed a positive correlation (Fig 1). The Spearman rank correlation coefficient $\left(r_{\mathrm{s}}\right)$ was $0.78(\mathrm{p}<0.001)$.

Visual assessment of 54 additional ${ }^{99 \mathrm{~m}} \mathrm{Tc}$ HMPAO scans was performed by three independent assessors. Total agreement in final visual score was obtained in only seven cases. There was a difference of one point in 17 cases, two points in 13 cases, three points in 11 cases, four points in five cases, and more than four points in two cases (one by five, one by seven). If a difference of two or less is regarded as reasonable agreement, then $69 \%$ of scans reached this level of agreement, but $31 \%$ failed to do so. Repeated scoring by one physician independently of the previous scores produced identical scores in $48 \%$, and a difference of two or less in $93 \%$. Repeated analysis by the computer of the scan score produced a coefficient of variation of only $5 \cdot 6 \%$.

The images from both types of tracer study (111 In and ${ }^{99 m}$ Tc HMPAO) could be used to localise the site of active disease. Previous studies have shown this to be accurate when compared with radiological, histological, and endoscopic assessment. ${ }^{4814}$ Figure 2 shows four representative pairs of images. The image quality in the ${ }^{99 m}$ Tc HMPAO scans was superior in every case, with sharper definition 
of the normal landmarks and the diseased bowel.

\section{Discussion}

Previous studies have independently verified the ability of ${ }^{111} \mathrm{In}$ and ${ }^{99 \mathrm{~m}}$ Tc HMPAO labelled leucocyte scintigraphy to localise intestinal inflammation in inflammatory bowel disease. ${ }^{4-6}$ It has also been shown that overall disease activity correlates well with the percentage of ${ }^{111}$ In excreted in a four day faecal collection. ${ }^{14}$ This has been used to measure the inflammatory response to treatment. ${ }^{15}$ Attempts to grade ${ }^{99 \mathrm{~m}}$ Tc HMPAO scans visually have been less successful, either because the grading system was too simple (zero to four) ${ }^{2}$ or, if more complicated, because it suffered unacceptable interobserver variation. ${ }^{16}$

In this study, the first to compare ${ }^{99 \mathrm{~m}} \mathrm{Tc}$ HMPAO scanning with faecal ${ }^{111}$ In excretion in a quantitative analysis, we have attempted to find out if reliable quantitative information can be obtained from ${ }^{99 m}$ Tc HMPAO scans. We have used both summated visual grading and computer derived scan scores. There is good correlation between percentage ${ }^{111}$ In faecal excretion and the $99 \mathrm{~m}$ Tc HMPAO scan score.

The failure of three patients to complete faecal collection is no worse than other centres, ${ }^{14}$ but illustrates an important drawback to ${ }^{111}$ In faecal assessment. Patients dislike faecal collection. Measurement of whole body ${ }^{111}$ In retention at four days has been proposed as an alternative to faecal collection. ${ }^{17}$ Unfortunately the four day delay in obtaining the result applies to both faecal collection and whole body retention and often prohibits the use of the investigation if the clinical problem is urgent.

The failure of one patient to excrete her ${ }^{111}$ In despite its appearance on the scan, because of drug induced constipation, shows that four day collection can be disrupted even in the most determined patients. Instructions on how to perform faecal collection need to be clear and explicit. The use of written details, as in this study, has been shown to minimise the discrepancy between ${ }^{111}$ In faecal collection and whole body ${ }^{111}$ In retention. ${ }^{17}$

One of the ${ }^{99 \mathrm{~m}} \mathrm{Tc}$ HMPAO scans showed an abnormal artefact that we cannot explain, but this is an unusual occurrence.

The summated visual grading assessment in this study showed an unacceptable interobserver variation with $31 \%$ of scans having significant disagreement between three observers. This level of variation would preclude its use for assessment of overall inflammation in therapeutic trials as well as reducing its value in routine clinical practice. The computerised scan score showed better reproducibility and therefore seems to overcome this problem. The subtle nature of the changes in activity in response to treatment has been shown by the use of faecal ${ }^{111}$ In excretion for this purpose, the percentage ${ }^{111}$ In excretion fell from the increased values in active disease but did not revert to the range for normal controls or fully inactive disease. ${ }^{1415}$
Both the ${ }^{111} \mathrm{In}$ and ${ }^{99 \mathrm{~m}} \mathrm{Tc}$ HMPAO techniques used mixed leucocytes rather than pure granulocytes. In the case of the ${ }^{111}$ In label pure granulocytes and mixed leucocytes have been compared previously. ${ }^{6}$ Pure granulocyte preparations seemed to produce scans with clearer images and may deliver a lower radiation dose to lymphocytes. Active and inactive Crohn's disease could be distinguished using either pure granulocytes or mixed leucocytes. ${ }^{6}$ Using mixed cells for ${ }^{111}$ In scanning necessitates an assumption of constant labelling efficiency for granulocytes. The percentage ${ }^{111}$ In labelling of pure granulocytes in a mixed cell preparation has previously been found to be $31 \%$ (SD $6 \%$ ). ${ }^{6}$ Ideally this percentage needs to be known for each patient, however, this requires a density gradient separation of granulocytes from an aliquot of mixed cells, a technique that requires additional expertise and facilities, which may not always be available. As ${ }^{99} \mathrm{~m}$ Tc HMPAO effectively labels granulocytes in a mixed cell preparation, further cell isolation procedures are unnecessary for this label. ${ }^{13}$

In this study the injected activities were on average ${ }^{111}$ In $15 \mathrm{MBq}$ and ${ }^{99 \mathrm{~m}}$ Tc HMPAO $200 \mathrm{MBq}$. The corresponding radiation doses have been calculated to be $6.75 \mathrm{cGy}$ and 4.0 cGy to the spleen, $0.9 \mathrm{cGy}$ and $0 \cdot 2 \mathrm{cGy}$ to the liver, and $1.65 \mathrm{cGy}$ and $0.25 \mathrm{cGy}$ to the bone marrow for ${ }^{111} \mathrm{In}$ and ${ }^{99 \mathrm{~m}}$ Tc HMPAO respectively. ${ }^{16}$ The radiation dosage is substantially lower with the ${ }^{99 \mathrm{~m}} \mathrm{Tc}$ HMPAO scanning technique, however, using ${ }^{99 \mathrm{~m} T c}$ HMPAO the image quality is superior to the ${ }^{111}$ In scans.

In conclusion, these results show that the ${ }^{99 \mathrm{~m} T c}$ HMPAO scan score has value in quantification of overall disease activity. This is similar to the percentage faecal ${ }^{111}$ In excretion with which it correlates closely. ${ }^{99 \mathrm{~m}} \mathrm{Tc} \mathrm{HMPAO}$ scans avoid faecal collection, produce superior images, and expose the patient to less radiation than ${ }^{111}$ In scintigraphy. ${ }^{99 \mathrm{~m}} \mathrm{Tc}$ HMPAO scans may therefore be the preferred method.

The authors wish to thank the staff of the Medical Physics Department at the Royal Hallamshire Hospital, especially Ms S Southern, for technical help performing the scans.

1 Saverymuttu SH, Peters AM, Hodgson HJ, Chadwick VS, Lavender JP. Indium-111 autologous leucocyte scanning: comparison with radiology for imaging the colon in comparison with radiology for imaging the colon
inflammatory bowel disease. $B M F 1982 ; 285: 255-7$.

2 Stein DT, Gray GM, Gregory PB, Anderson M, Goodwin DA, McDougall IR. Location and activity of ulcerative and Crohn's colitis by indium 111 leukocyte scan. Gastroenterology 1983; 84: 388-93.

Crama-Bohbouth GE, Peña AS, Arndt JW, Tjon RTO Tham A, Verspaget HW, et al. Value of indium-111 ropolonate autologous granulocyte scintigraphy in the assessment of inflammatory bowel disease. Scand f Gastroenterol 1990; 25 (suppl 178): 93-8.

4 Schölmerich J, Schmidt E, Schümichen C, Billmann $P$ Schmidt H, Gerok W. Scintigraphic assessment of bowel involvement and disease activity in Crohn's disease using technetium $99 \mathrm{~m}$-hexamethyl propylene amine oxine as technetium 99m-hexamethyl propylene amine oxine

5 Vilien M, Nielsen SL, Jørgensen M, Binder V, Hvid Jacobson $\mathrm{K}$, Berild $\mathrm{D}$, et al. Leucocyte scintigraphy to localize inflammatory activity in ulcerative colitis and Crohn's disease. Scand f Gastroenterol 1992; 27: 582-6. Crohn's disease. Scand F Gastroenterol 1992; 27: 582-6.
Saverymuttu SH, Peters AM, Lavender JP, Pepys MB, Saverymuttu SH, Peters AM, Lavender JP, Pepys MB,
Hodgson HJF, Chadwick VS. Quantitative fecal indium 111-labelled leukocyte excretion in the assessment of disease in Crohn's disease. Gastroenterology 1983; 85: 1333-9.

7 Almer S, Franzén L, Peters AM, Tjädermo M, Ekberg S, Granerus G, et al. Do technetium-99m hexamethylpropylene amine oxime-labeled leukocytes truly reflect the mucosal inflammation in patients with ulcerative colitis? Scand $\mathcal{F}$ Gastroenterol 1992; 27: 1031-8. 
8 Sciaretta G, Furno A, Mazzoni M, Basile C, Malaguti P. Technetium- $99 \mathrm{~m}$ hexamethyl propylene amine oxime granulocyte scintigraphy in Crohn's disease: diagnostic granulocyte scintigraphy in Crohn's disease:
and clinical relevance. Gut 1993; 34: 1364-9.

9 Becker W, Fischbach W, Kirchner T, Reiners C, Börner W. Leukozytenszintigraphie als Aktivitätsparameter bei Morbus Crohn. Z Gastroenterol Vehr Bd 1987; 22: 178-84

10 Tindale WB, Barber DC, Giaffer MH, Senior S, Holdsworth $C D$. ${ }^{99} \mathrm{~m}$ Tc HMPAO-labelled leucocyte imaging in Crohn's disease: a subtraction technique for the quantification of disease activity. Clin Phys Physiol Meas 1992; 13: 37-50.

11 Giaffer $\mathrm{MH}$, Tindale WB, Senior S, Barber DC, Holdsworth CD. Quantification of disease activity in Crohn's disease by computer analysis of Tc-99m hexamethyl propylene amine oxime (HMPAO) labelled leucocyte images. Gut 1993; 34: 68-74.

12 Saverymuttu SH, Peters AM, Danpure HJ, Reavy HJ, Osman S, Lavender JP. Lung transit of ${ }^{111}$ indium-labelled granulocytes. Scand $\mathcal{F}$ Haematol 1983; 30: 151-60.

13 Danpure HJ, Osman S, Carroll MJ. The development of a clinical protocol for the radiolabelling of mixed leucocytes with ${ }^{99 \mathrm{~m}}$ Tc-hexamethylpropyleneamine oxime. Nucl Med Wommun 1988; 9: $465-75$

14 Saverymuttu SH, Camilleri M, Rees H, Lavender JP, Hodgson HJF, Chadwick VS. Indium 111-granulocyte scanning in the assessment of disease extent and disease activity in inflammatory bowel disease. Gastroenterology 1986; 90: 1121-8.

15 Becker W, Fischbach W, Weppler M, Mosl B, Jacoby G, Börner W. Radiolabelled granulocytes in inflammatory bowel disease: diagnostic possibilities and clinical indications. Nucl Med Commun 1988; 9: 693-701.

16 Teahon K, Smethurst P, Pearson M, Levi AJ, Bjarnason I. The effect of elemental diet on permeability and inflammation in Crohn's disease. Gastroenterology 1991; 101: 84-9.

17 Carpani de Kaski M, Peters AM, Knight D, Stuttle AWJ, Lavender $\mathrm{P}$, Hodgson $\mathrm{HJ}$. Indium-111 whole body retention: a method for quantification of disease activity in inflammatory bowel disease. F Nucl Med 1992; 33: 756-62. 